\title{
Integrated Intersection Evaluation Method Based on BP Neural Network
}

\author{
Yan Zhang ${ }^{\text {a }}$, Yanxin Zhang ${ }^{\text {b }}$ \\ School of Electronic and Information Engineering, Beijing Jiao Tong University, Beijing 10044, \\ China. \\ a16120279@bjtu.edu.cn, byxzhang@bjtu.edu.cn
}

\begin{abstract}
With the development of the Wangjing regional economy, the number of cars in the region has increased. In order to allow inter-regional vehicles to pass with high efficiency, it is necessary to perform some efficient control at the boundary of the area. First, controlling the intersection of the entire transportation network is not effective and economical. Therefore, it is necessary to evaluate the boundary intersections so as to select the more important intersections on the regional boundaries for control.In this paper, the road network partition was first carried out. This paper mainly uses the basic properties of the traffic network to partition the road network. The boundary intersections are evaluated based on the repartitioning to select more important intersections for control at the regional boundaries. In this paper, we mainly use the three indicators of social network analysis method, system science analysis method, and traffic network characteristics method to evaluate the intersections, and use neural network training to get the weight of each indicator, so as to determine a comprehensive evaluation method. Feedback gate control based on fuzzy PID is applied to the traffic network based on the selected important intersection, thereby alleviating the congestion in the effective central city.
\end{abstract}

Keywords: Transportation network, Social Network Analysis busyness, Systematic scientific analysis, Traffic characteristics, Neural network.

\section{Introduction}

As the number of vehicles in the Wangjing area has increased, the probability of traffic jams and accidents has also gradually increased. Traffic jams are mainly due to irrational signal timing at intersections and limited capacity. In order to reduce the complexity of signal control between regions, it has become a mainstream trend to select important intersections at regional boundaries and control these intersections[1-9]. As a result, the throughput of the vehicles in the area is optimized. The previous major intersection studies were mainly divided into three categories. In the literature[10, 11], the degree of centrality in social network analysis was used to evaluate the importance of nodes. In the literature[12-14]was used the system science analysis method to evaluate the nodes, which are the node contraction method, the node deletion method, and the m-order neighbor node method to evaluate the nodes. The node contraction method is fast and can evaluate the importance of complex network nodes with high efficiency, but it does not combine the characteristics of traffic network, so it can not scientifically and effectively evaluate the importance of intersections in traffic networks. The m-order neighbor node fully considers the influence of the neighbor node on this node. The criterion of node deletion method is the degree of network change after node deletion. In the literature[15-18] evaluates the urban road network by adding traffic flow characteristics in the road network, such as: the popularity of the route, the speed of the trip, and the density of traffic, etc., to determine the importance of the intersection. At present, the method of using social network analysis method and system science method to evaluate nodes in complex networks has become more mature. Based on this, the attributes of traffic network are added and neural network is used to obtain a comprehensive evaluation index with higher credibility. 


\section{Important Assessment Methods}

This paper mainly selects four indicators (centrality, node contraction method, busyness, congestion degree) from above three aspects to determine the importance of nodes in the transportation network.. The specific indicators are as follows:

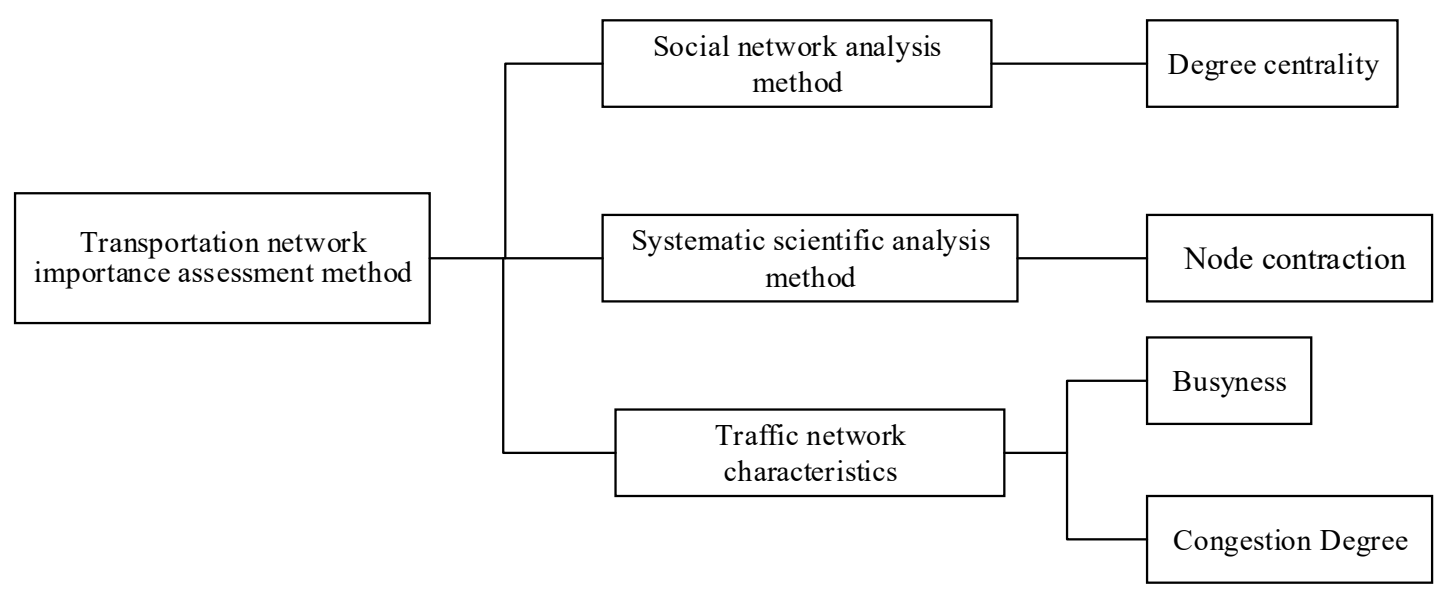

Fig.1 Evaluating indicator

\subsection{Centrality}

In the Social network analysis method, the degree of a node is the number of nodes adjacent to the node in a complex network, is defined as follows.

$$
D C(i)=\frac{\sum a_{i j}}{n-1}=\frac{k_{i}}{n-1}
$$

In the formula: $a_{i j}$ is the element in the adjacency matrix of the network, $\sum a_{i j}$ is the number of connected nodes of node $i, k_{i}$ is the degree of node $i$ in the network, and $n$ is the number of nodes in the complex network.

From the difination, we know that the greater the number of node neighbors is, the more important the nodes are. It can directly reflect the influence of nodes, which should be the most important characteristics in the complex network.

\subsection{The Node Contraction Method}

From Systematic scientific analysis, the node contraction method is to Shrink the node with the node to 1 node, that is, to generate one new node to replace nodes.

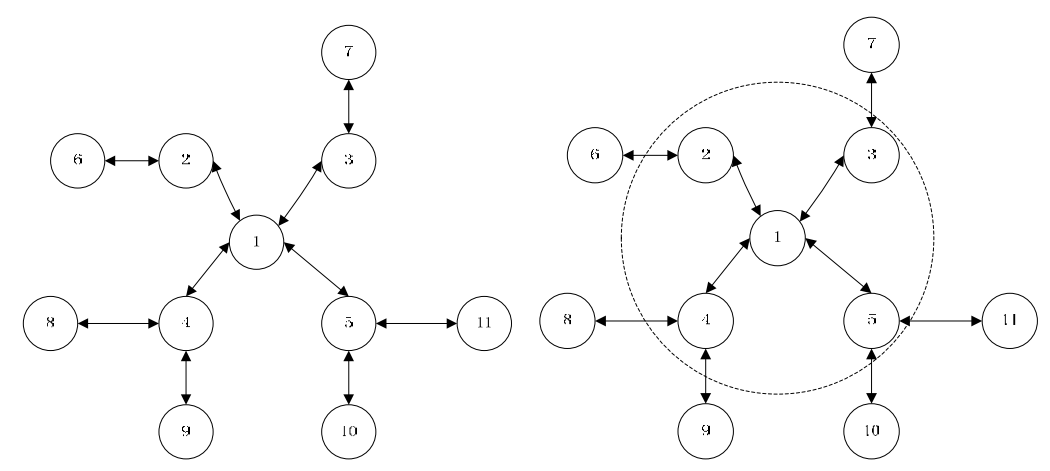

Fig.2 meshed network

For the degree of aggregation of network, it is defined as: 


$$
\partial[G]=\frac{1}{n \times l}=\frac{n-1}{\sum_{i \neq j} d_{i j}}
$$

Where $n$ is the number of nodes, $l$ is the average path length, and $d_{i j}$ is the shortest distance between nodes $i$ and $j$.

The evaluation of the importance of the node $v_{i}$ in the network $G$ is mainly combined with the degree of aggregation before and after the contraction of the node $v_{i}$, and defined as:

$$
\operatorname{IMC}\left(v_{i}\right)=1-\frac{\partial[G]}{\partial\left[G * v_{i}\right]}
$$

In the formula: $\partial[G]$ is the degree of aggregation of the network, and $\partial\left[G * v_{i}\right]$ is the degree of aggregation of the new network resulting from the fusion of node $v_{i}$ with the surrounding $k_{i}$ nodes. It is proved that nodes $k_{i}$ are important nodes in a complex network, if the network has the highest degree of aggregation.

\subsection{Traffic Network Characteristics}

\subsubsection{Busyness}

The busyness is obtained from the traffic volume of the current road section and the saturated traffic volume of the road section and is defined as:

$$
F_{i}=\frac{q_{i}}{c_{i}}
$$

Where $F_{i}$ is the busyness of the intersection, $q_{i}$ is the traffic flow, and $c_{i}$ is the saturation flow.

\subsubsection{Congestion Degree}

It is shown that the amount of fuel consumed by the same number of vehicles is different at different times even for a certain road segment. During periods of congestion, the vehicle consumes a large amount of fuel because of its start-up and braking. Although some road junctions in the urban traffic network are relatively busy, the intersections are not easy to be congested due to the large capacity of the roads. Therefore, this paper proposes to use fuel consumption as an evaluation index. In addition to the topological attributes of the road network and the busyness of intersections, a new indicator is added. In order to use a variety of factors to evaluate the intersection, we can scientifically and effectively judge the importance of the intersection in the road network. The degree of congestion at the intersection is based on the fuel consumption of the current intersection and the total fuel consumption of the area. The data in this paper is provided by the Intelligent Transportation Center, where fuel consumption is collected by the fuel consumption sensors. The degree of congestion is as follows:

$$
Y_{i}=\frac{O_{i}}{O}
$$

Where $o_{i}$ is the fuel consumption at the intersection and $O$ is the total fuel consumption of the area. 


\section{Comprehensive Evaluation Model}

The nodal shrinkage method has the characteristics of being intuitive, effective, and faster, and has an ideal computing capability in a complex network. However, in the transportation network, it is obviously not enough to consider only the characteristics of the network topology. Therefore, traffic flow and fuel consumption are included in the assessment index. The traffic flow can be used to evaluate the busyness of the road section. The fuel consumption is used to evaluate the number of vehicles parking in a certain road section and the degree of congestion on the road section. The centrality determines the influence of the intersection through the number of intersections that are connected to the intersection. As a result, a comprehensive assessment indicator is produced as follows:

$$
I\left(v_{i}\right)=\alpha \operatorname{IMC}\left(v_{i}\right)+\beta F\left(v_{i}\right)+\mu Y\left(v_{i}\right)+\delta \operatorname{IMD}\left(v_{i}\right)
$$

In the formula: $I\left(v_{i}\right)$ is the degree of importance of the nodes in the transportation network, $\operatorname{IMC}\left(v_{i}\right)$ is the degree of importance obtained by the node contraction method, $\operatorname{IMD}\left(v_{i}\right)$ is the degree of importance obtained by using the degree of the node, $F\left(v_{i}\right)$ is the busy degree, and $Y\left(v_{i}\right)$ is the degree of congestion. $\alpha, \beta, \mu, \delta$ is the weight of different assessment indicators, which can be trained by neural networks.

In order to determine the weights of various assessment indicators in the transportation network, neural network technology is used here, which needs known sample learning and expert experience[19]. The following figure is a basic BP (Back-Propagation) neuron model, which has four inputs as $i$,one-output as $j$, connected by corresponding weights and transfer functions, the output of the network is:

$$
Y=f(\omega X+\theta)
$$

Where $x_{1}$ is the degree of each node, $x_{2}$ is the importance of each node obtained by the node contraction method, $x_{3}$ is the busyness of the intersection, and $x_{4}$ is the congestion degree of the intersection. This article mainly uses the busyness of intersections in different time periods to train the weight value closest to the expected value.

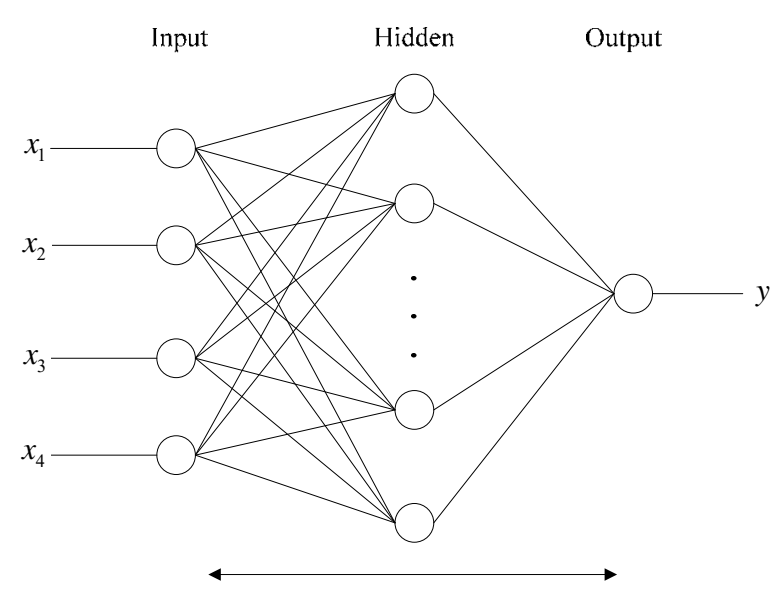

Fig.3 back propagation neural network

The input from the hidden layer is: 


$$
N e t_{k}^{1}=\sum_{i=1}^{m} w_{k i}^{1} x_{i}+\theta_{k}
$$

Where $w_{k i}^{1}$ is the weight of the first layer, and $x_{i}$ is the value of the $i$ input of the neural network, and the number of elements in the input layer is $m, \theta_{k}$ is the threshold of the hidden layer. Hidden layer output:

$$
o_{k}=f\left(N e t_{k}^{1}\right)=f\left(\sum_{i=1}^{m} w_{k i}^{1} x_{i}+\theta_{k}\right)
$$

In the formula: $o_{k}$ is the output of the hidden layer and $f$ is the transfer function.

Transfer function (S-function):

$$
f(x)=\frac{1}{1+e^{-x}}
$$

Output layer input:

$$
N e t_{o}^{2}=\sum_{k=1}^{p} w_{o k}^{2} o_{k}+\theta_{o}
$$

In the formula: $o_{k}$ is the output of the hidden layer, $w_{o k}^{2}$ is the second layer weight, $p$ is the number of neurons in the hidden layer, and $\theta_{o}$ is the output layer threshold.

Output layer output:

$$
y_{o}=f\left(N e t_{o}^{2}\right)=f\left(\sum_{k=1}^{p} w_{o k}^{2} o_{k}+\theta_{o}\right)
$$

Hidden layer to output layer weight correction:

$$
\Delta w_{o k}^{2}=\eta\left(\widehat{y_{o}}-y_{o}\right) y_{o}\left(1-y_{o}\right) o_{k}
$$

The correction of the weight of the input layer to the hidden layer:

$$
\Delta \omega_{k i}^{1}=-\eta \sum_{i=1}^{n}\left[\left(\widehat{y}_{o}-y_{o}\right) y_{o} \omega_{o k}^{2}\right] o_{k}\left(1-o_{k}\right) x_{i}
$$

By using the BP neural network method, input data including the node contraction method, busy degree, congestion degree, and degree-centrality data into the $\mathrm{NN}$ for training, and obtain the weight matrix from the input layer to the hidden layer, the hidden layer to the output layer. From the weight matrix, we can get the weight of each index.

\section{Experimental Analysis}

\subsection{Establishment of Beijing Wangjing Regional Road Network Model}

The Beijing Wangjing area is located in the northeast of Beijing. Large traffic volume at intersections and limited traffic capacity can easily cause traffic congestion. Therefore, it is necessary to screen out important nodes through the method of importance evaluation, allocate more traffic 
resources to these important nodes and choose the optimal signal timing plan, so as to ensure the smooth operation of traffic at this these intersections. This experiment selected some areas of Wangjing(longitude: 116.47014141-116.48400307, latitude:39.9877436-39.99875571:). As shown below.

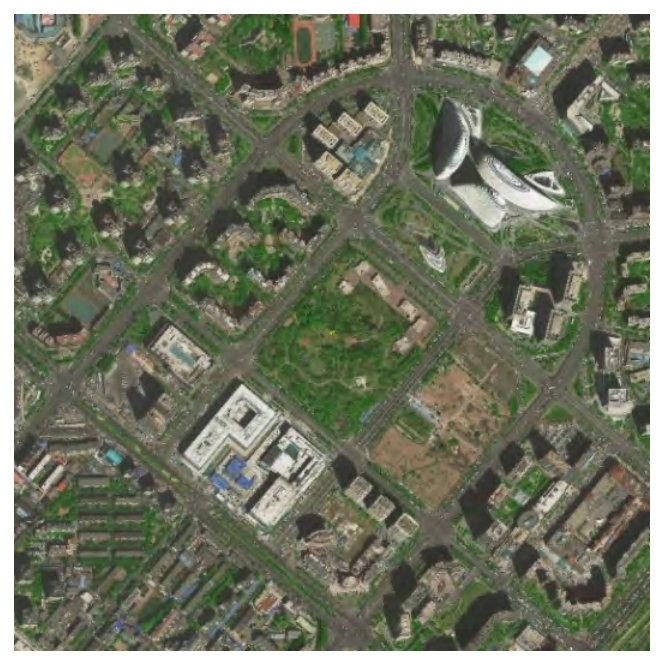

Fig.4 Selected areas under the Google map

\subsection{Simulation Data Acquisition}

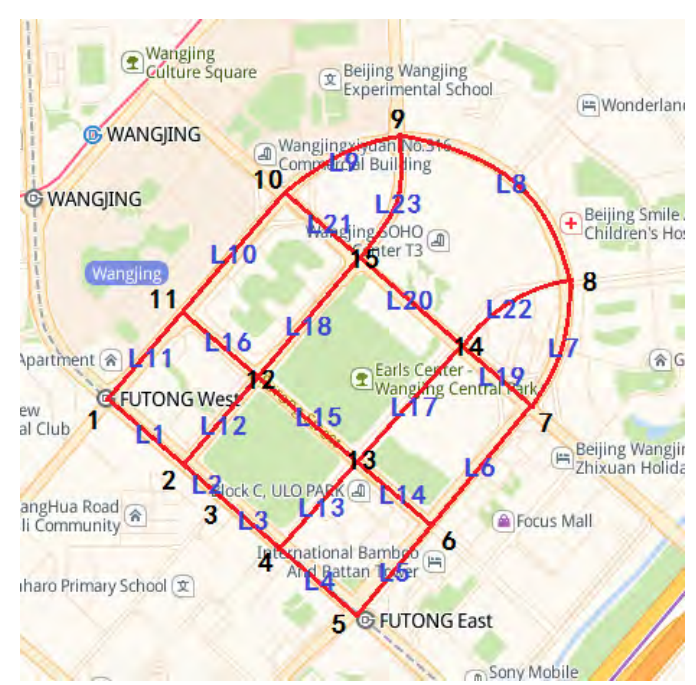

Fig.5 Wangjing region

First, we use the Gaode map to find the selected area, and then number the intersections and sections in the selected Wangjing area (see Figure 15). There are 15 intersections and 23 sections in this area. Based on the longitude and latitude of the Wangjing area, the floating car data in Beijing were screened, and then the traffic data, fuel consumption, steering angle, etc. of the floating cars in the Wangjing area were processed. Then the length of each road section was measured by the distance measurement function of the Baidu map. The specific data is shown in the table below. Table 1. Road length

\begin{tabular}{cccccc}
\hline Road & Length & Road & Length & Road & Length \\
\hline L1 & 215 & L9 & 280 & L17 & 355 \\
L2 & 103 & L10 & 350 & L18 & 360 \\
L3 & 160 & L11 & 250 & L19 & 213 \\
L4 & 210 & L12 & 255 & L20 & 280 \\
L5 & 255 & L13 & 250 & L21 & 295 \\
L6 & 360 & L14 & 200 & L22 & 308 \\
L7 & 305 & L15 & 280 & L23 & 295 \\
L8 & 550 & L16 & 210 & & \\
\hline
\end{tabular}


The data used in this paper is the Beijing taxi data provided by the Intelligent Traffic Big Data Center. Through the latitude and longitude of the study area, the floating car data of the selected area are screened out in the data of the total floating car, such as vehicle identification, instantaneous fuel consumption, etc. The main data is shown in the table below.

Table 2. Floating car data

\begin{tabular}{cccccc}
\hline Intersection & Number & Fuel & Intersection & Number & Fuel \\
\hline V1 & 3796 & 900953 & V9 & 506 & 132910 \\
V2 & 1773 & 470000 & V10 & 3289 & 815690 \\
V3 & 1549 & 357421 & V11 & 420 & 114091 \\
V4 & 837 & 219822 & V12 & 1664 & 510998 \\
V5 & 3565 & 884747 & V13 & 2450 & 600218 \\
V6 & 2413 & 558561 & V14 & 1550 & 412694 \\
V7 & 2622 & 652883 & V15 & 1276 & 370365 \\
V8 & 335 & 91458 & & & \\
\hline
\end{tabular}

\subsection{Importance Evaluation Method Simulation}

Using the node contraction method to assess the importance of the intersection in the Wangjing area:

1) Enter the initial distance matrix and calculate the shortest distance between the nodes.

2) The pre-shrinkage nodal agglomeration is calculated by the formula.

3) In the following figure, node 1 is shrunked. The degree of condensation after shrinkage is calculated, and the importance of the node is obtained by the degree of aggregation before and after the contraction of the node.

4) Calculate the aggregation of each node in turn.Get the importance of all nodes and sort them.

The schematic diagram of the node contraction method applied in the Wangjing area is as follows:

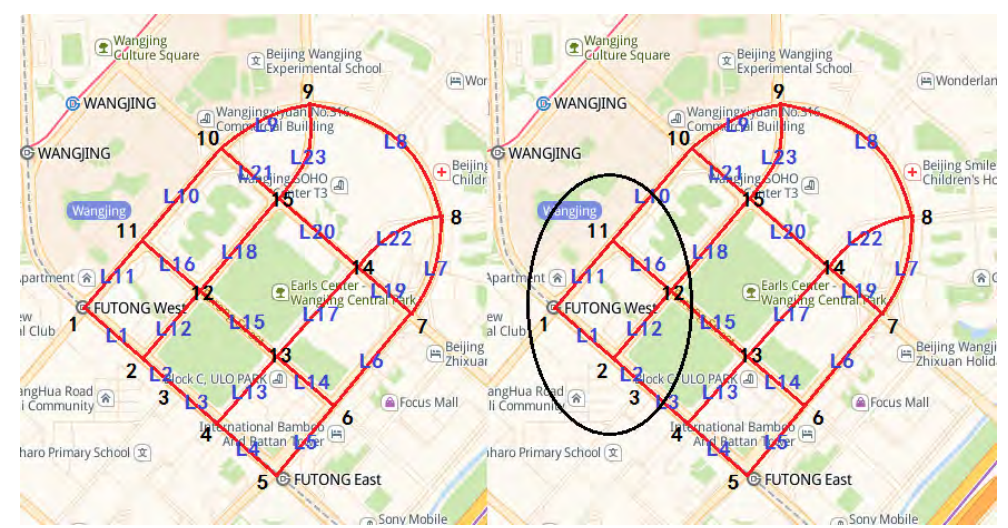

Fig.6 Node contraction

Using the degree of business, degree of centrality, node contraction method and the fuel consumption of the vehicles to get the importance of the following table.

Table 3. The importance of the nodes obtained by the various factors

\begin{tabular}{cccccccccc}
\hline Intersection & Busyness & Congestion & degree & IMC & Intersection & Busyness & Congetion & degree & IMC \\
\hline V1 & 0.135 & 0.127 & 0.143 & 0.561 & V9 & 0.018 & 0.018 & 0.214 & 0.626 \\
V2 & 0.063 & 0.066 & 0.214 & 0.592 & V10 & 0.117 & 0.115 & 0.214 & 0.614 \\
V3 & 0.055 & 0.050 & 0.143 & 0.559 & V11 & 0.015 & 0.016 & 0.214 & 0.603 \\
V4 & 0.030 & 0.031 & 0.214 & 0.592 & V12 & 0.059 & 0.072 & 0.286 & 0.604 \\
V5 & 0.127 & 0.125 & 0.143 & 0.561 & V13 & 0.087 & 0.085 & 0.286 & 0.605 \\
V6 & 0.086 & 0.079 & 0.214 & 0.605 & V14 & 0.055 & 0.058 & 0.286 & 0.647 \\
V7 & 0.093 & 0.092 & 0.214 & 0.616 & V15 & 0.045 & 0.052 & 0.286 & 0.643 \\
V8 & 0.012 & 0.013 & 0.214 & 0.524 & & & & & \\
\hline
\end{tabular}


Judging by the degree of congestion, V1 intersection is the most important intersection. The use of node contraction method to determine the V14 intersection as the most important intersection. When the centrality of degree is used, it is determined that V12, V13, V14, and V15 are the most important intersections. Different evaluation factors have got different results. Therefore, we need to obtain the weights of different evaluation factors through neural network training, taking into account the influence of various factors on the intersections, and thus scientifically and effectively determine the importance of intersections.

\subsection{Neural Network Simulation}

Experiments have shown that when the number of neurons in the hidden layer is 8 , the convergence is better at this time and the calculation is smaller.This article selected some samples to be trained through a neural network. The weights obtained from the BP neural network are shown in the following table.

Table 4. Weight coefficient table of four evaluation indexes of traffic network

\begin{tabular}{cccccc}
\hline \multirow{2}{*}{ Hidden layer unit } & \multicolumn{4}{c}{ Input layer unit } & \multirow{2}{*}{ Output layer unit } \\
\cline { 2 - 4 } & 1 & 2 & 3 & 4 & \\
\hline V1 & -1.459 & -0.999 & -0.960 & 1.260 & -0.142 \\
V2 & -0.258 & 1.504 & -1.515 & 0.971 & -0.145 \\
V3 & -1.439 & -1.067 & 1.032 & 1.135 & 0.208 \\
V4 & 0.887 & 1.664 & -0.652 & 1.178 & -0.278 \\
V5 & -0.830 & -1.768 & 1.107 & -0.670 & 0.323 \\
V6 & 2.058 & -0.678 & -0.512 & 0.874 & 1.352 \\
V7 & 1.062 & -2.008 & 0.608 & 0.354 & -0.616 \\
V8 & 0.323 & 0.649 & -2.206 & 0.167 & 0.353 \\
\hline
\end{tabular}

The weights of the input layer-hidden layer and the hidden layer-output layer can be obtained through the neural network. However, we ultimately need to obtain the weight relationship of degreecentrality, busyness, fuel consumption, and node contraction method. Therefore, the following indicators are needed.

Relevant significant coefficient

$$
\begin{gathered}
r_{i o}^{T}=\sum_{k} \omega_{k i}\left(1-e^{-x}\right) /\left(1+e^{-x}\right) \\
x=\omega_{j k}
\end{gathered}
$$

Correlation coefficient

$$
\begin{gathered}
R_{\text {io }}=\left|\left(1-e^{-y}\right) /\left(1+e^{-y}\right)\right| \\
y=r_{\text {io }}
\end{gathered}
$$

Absolute influence coefficient

$$
S_{i o}=R_{i o} / \sum_{i=1}^{m} R_{i o}
$$

In the formula: $w_{k i}$ is the weight matrix between the input layer and the hidden layer, $w_{j k}$ is the weight matrix between the hidden layer and the output layer, $m$ is the number of input layer nodes, $n$ is the number of output layer nodes, $p$ is the hidden layer node number. 
Calculated by MATLAB: $\alpha=0.6570, \beta=0.0973, \mu=0.1131, \delta=0.0503$

The order of the importance of the final node is shown in the following table.

Table 5. Node importance ranking

\begin{tabular}{cccccc}
\hline Intersection & Importance & Rank & Intersection & Importance & Rank \\
\hline V1 & 0.306 & 13 & V9 & 0.540 & 6 \\
V2 & 0.406 & 10 & V10 & 0.698 & 3 \\
V3 & 0.098 & 15 & V11 & 0.363 & 11 \\
V4 & 0.320 & 12 & V12 & 0.526 & 8 \\
V5 & 0.299 & 14 & V13 & 0.574 & 5 \\
V6 & 0.538 & 7 & V14 & 0.819 & 1 \\
V7 & 0.654 & 4 & V15 & 0.777 & 2 \\
V8 & 0.514 & 9 & & & \\
\hline
\end{tabular}

From the above table, it can be seen that the major intersections V14, V15, and V10 are evaluated using the comprehensive evaluation index, and the less important intersections are V1, V5, and V3. Using comprehensive assessment indicators, the impact of each factor on the intersection can be analyzed more comprehensively and clearly, making the assessment result more reasonable and credible.

\section{Conclusion}

This paper takes the typical region of Wangjing in Chaoyang District of Beijing as a model. With a view to allocate more traffic resources to the more important intersections in the actual road network in order to avoid crossroads, it is more conducive to choose the most important intersections. Firstly, the limitations of some traditional methods for assessing the importance of nodes in complex networks are analyzed. Secondly, a comprehensive index for assessing the importance of intersections was established by increasing the busyness, the degree of congestion obtained from fuel consumption, the degree of centrality, node contraction method. And using BP neural network training to get the weight of each factor in this comprehensive evaluation index, from the perspective of mathematical analysis of the importance of the intersection. The redistribution of road resources at the important intersections selected this time can effectively reduce the pressure at the intersections.

\section{Acknowledgments}

Fund project: The Fundamental Research Funds for the Central Universities (No. W16JB00340).

\section{References}

[1]. Ye B, Wu W, Li L, et al. A Hierarchical Model Predictive Control Approach for Signal Splits Optimization in Large-Scale Urban Road Networks[J]. 2016,17(8):2182-2192.

[2]. Feng Y, Head K L, Khoshmagham S, et al. A real-time adaptive signal control in a connected vehicle environment[J]. Transportation Research Part C: Emerging Technologies, 2015,55:460473.

[3]. Sirmatel I I, Geroliminis N. Economic Model Predictive Control of Large-Scale Urban Road Networks via Perimeter Control and Regional Route Guidance[J]. IEEE T INTELL TRANSP, 2018,19(4):1112-1121.

[4]. $\mathrm{Fu} \mathrm{H}, \mathrm{Liu} \mathrm{N}, \mathrm{Hu} \mathrm{G}$. Hierarchical perimeter control with guaranteed stability for dynamically coupled heterogeneous urban traffic[J]. Transportation Research Part C: Emerging Technologies, 2017,83:18-38. 
[5]. Keyvan-Ekbatani M, Papageorgiou M, Knoop V L. Controller design for gating traffic control in presence of time-delay in urban road networks[J]. Transportation Research Part C: Emerging Technologies, 2015,59:308-322.

[6]. Keyvan-Ekbatani M, Kouvelas A, Papamichail I, et al. Exploiting the fundamental diagram of urban networks for feedback-based gating[J]. Transportation Research Part B: Methodological, 2012,46(10):1393-1403.

[7]. Ji Y, Mo C, Ma W, et al. Feedback Gating Control for Network Based on Macroscopic Fundamental Diagram[J]. MATH PROBL ENG, 2016,2016:1-11.

[8]. Fu H, Liu N, Hu G. Hierarchical perimeter control with guaranteed stability for dynamically coupled heterogeneous urban traffic[J]. Transportation Research Part C: Emerging Technologies, 2017,83:18-38.

[9]. Zhou Z, De Schutter B, Lin S, et al. Two-Level Hierarchical Model-Based Predictive Control for Large-Scale Urban Traffic Networks[J]. IEEE T CONTR SYST T, 2017,25(2):496-508.

[10]. Jin J, Xu K, Xiong N, et al. Multi-index evaluation algorithm based on principal component analysis for node importance in complex networks[J]. 2012,1(3):108.

[11]. Ren Xiaolong, Lv Linyuan. A Survey of Ranking Methods for Important Nodes in Network[J]. Chinese Science Bulletin, 2014(13):1175-1197.

[12]. Ji Yangbeibei, Winnie Daame. Research on Layout Method of Urban Road Coil Detector in Amsterdam[J]. Journal of Chongqing Jiaotong University (Natural Science Edition), 2010(05):754-757.

[13]. Tan Yuejin, Wu Jun, Deng Hongzhong. Node contraction method for node importance evaluation in complex networks[J]. System Engineering Theory and Practice, 2006(11):79-83.

[14]. Zhang Xiping, Li Yongshu, Liu Gang. Complex network node importance evaluation method based on node importance contribution[J]. Complex systems and complexity science , 2014(03):26-32.

[15]. Hong zenglin, Liu Bingyan, Zhang Yapei. Application of Complex Network in Evaluation of Traffic Network Node Importance[J]. Journal of Xi'an University of Technology , 2014(05):404-410.

[16]. Wang Jianqiang, Dai Leilei. Method for distinguishing key intersections of urban trunk lines based on traffic flow characteristics[J]. Traffic information and security, 2013(03):49-52.

[17]. Xu Ming, Wu Jianping, Du Yiman. Road network node key degree ranking method based on three-part graph[J]. Journal of Beijing University of Posts and Telecommunications, 2014,37(z1):51-54.

[18]. Cai Jianrong, Research on the Evaluation Method of Traffic Network Node Importance and Its Application[D]. Changsha University of Science and Technology, 2014.59.

[19]. Sun Huijun, Wang Xinhua. Application of artificial neural network to determine the weight of evaluation indicators[J]. Journal of Shandong University of Science and Technology (Natural Science Edition), 2001(03):84-86. 